\title{
Narrow-band source of polarization-entangled photons with counterpropagating domain engineering
}

\author{
Yi-Chen Liu ${ }^{1}$, Dong-Jie Guo ${ }^{1}$, Zhen-da Xie ${ }^{1+}$, Yan-Xiao Gong ${ }^{1+}$ and Shi-Ning Zhu ${ }^{1}$ \\ ${ }^{1}$ National Laboratory of Solid-State Microstructures, Collaborative Innovation Center of Advanced \\ Microstructures, Nanjing University, Nanjing, China 210093 \\ *E-mail: xiezhenda@nju.edu.cn,gongyanxiao@nju.edu.cn
}

\begin{abstract}
Photonic entanglement is central resource to quantum information sciences, such as quantum communication and quantum computation. The entangled photons generated in conventional spontaneous parametric down-conversion usually yield $\mathrm{THz}$ bandwidth which becomes very dim in many applications call for narrow-band entanglement sources. Here we demonstrate the polarization-entanglement photon source with counterpropagating phase matching, which results in an inherent bandwidth of $7.1 \mathrm{GHz}$ at telecom wavelength. The entanglement is measured to violate the Bell inequality by up to 18.5 standard deviations, with Clauser-Horne-Shimony-Holt S-parameter of $2.720 \pm 0.039$. The quantum state tomography further characterizes the entanglement, with fidelity $\mathrm{F}=(95.71 \pm 0.61) \%$.
\end{abstract}

The bandwidth of entangled light source which plays a central role in quantum information is a crucial characteristic that determines its application prospect. That's because the interaction between light and matter, which is at the heart of many applications of quantum information, requires a narrow-band light source. This restrict has been loosed to $\mathrm{GHz}$ in the last few years ${ }^{[1,2]}$. Even though, to be honest, it is still a challenge for conventional spontaneous parametric down-conversion which yield a bandwidth about THz. Hence, for the first time, we proposed a state-of-art domain-engineering technology for the counterpropagating phase matching in the polarization-entanglement generation.
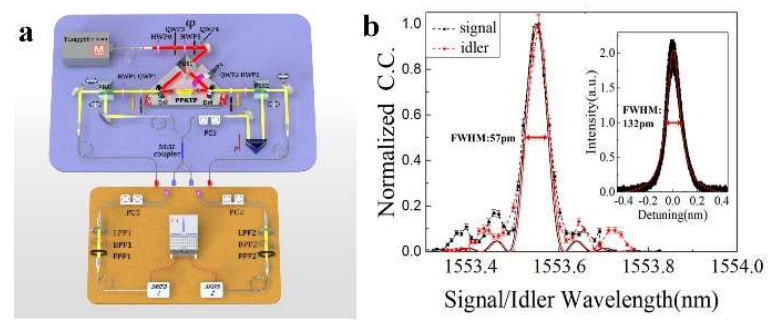

Fig. 1. a. The experimental setup of the preparation and representation of the photons. b. The spectrum of the down-converted photons.

In order to representation the bandwidth of the photon source, the spectral of signal and idler has been proceeded by measuring the coincidence counts after spectral filtering with a result of $57 \mathrm{pm}(7.1 \mathrm{GHz})$. The Hong-Ou-Mandel (HOM) interference has been measured with a base-to-base width of 155 ps and a visibility of $97.1 \%$. The visibilities of polarization correlation measurement are $(96.6 \pm 0.45) \%,(99.5 \pm$
$0.19) \%, \quad(97.1 \pm 0.53) \%$ and $(97.2 \pm 0.53) \%$, respectively. The quantum state tomography further characterizes the entanglement, with fidelity $\mathrm{F}=(95.71 \pm$ $0.61) \%$.
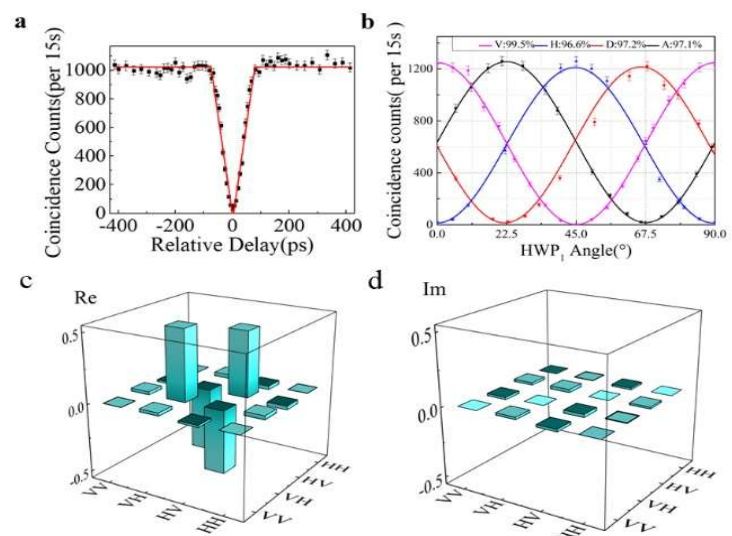

d

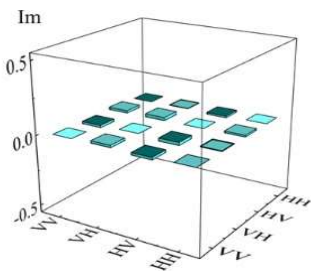

Fig. 2. a. HOM interferometer. b. Entanglement correlation measurement. c.d. The real (c) and imaginary (d) parts of the reconstructed density matrix for the polarization states

\section{Reference}

[1] J. Jin et al. "Telecom-wavelength atomic quantum memory in optical fiber for heralded polarization qubits," Phys. Rev. Lett. 115(14), 140501-140505 (2015).

[2] E. Saglamyurek et al. "Broadband waveguide quantum memory for entangled photons," Nature 469(7331), 512-515 (2011). 